the equation $\sum_{m=0}^{s} A_{m} \cdot \times K_{m} X^{m}=0$, if the matrices involved are square.

Results similar to those in this paper may be obtained, in some cases more readily, in the consideration of $\sum_{m=0}^{s}\left(X^{m} K_{m}\right) \cdot \times A_{m}=0$, $\sum_{m=0}^{s} A_{m} X \cdot\left(K_{m} X^{m}\right)=0$, and $\sum_{m=0}^{s}\left(X^{m} K_{m}\right) X \cdot A_{m}=0$, and so on, where $A \times \cdot B=\left(a_{i j} B\right)$.

\title{
BIBLIOGRAPHY
}

1. M. H. Ingraham, Rational methods in matrix equations, Bull. Amer. Math. Soc. vol. 47 (1941) pp. 61-70.

2. C. C. MacDuffee, The theory of matrices, Ergebnisse der Mathematik und ihrer Grenzgebeite, Chelsea, 1946, pp. 31-36.

3. J. H. Bell, Left associates of monic matrices with an application to unilateral matrix equations, Amer. J. Math. vol. 71 (1949).

Michigan State College

\section{AVERAGES OF CHARACTER SUMS}

\section{P. T. BATEMAN AND S. CHOWLA}

Suppose that $\chi$ is a primitive residue character ${ }^{1}$ modulo $k, k>1$,

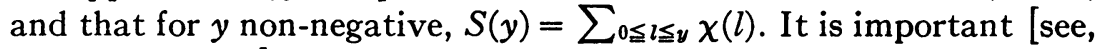
for example, 11] in the analytic theory of numbers to have as much information as possible about the sums $S(y)$, in particular about their maximum order of magnitude; it is known (cf. $[13 ; 14 ; 8]$ ), for example, that $S(y)<k^{1 / 2} \log k$, but unknown whether or not $M(\chi)$ $=o\left(k^{1 / 2} \log k\right)$ as $k$ tends to infinity, where $M(\chi)$ is the maximum of $|S(1)|, \cdots,|S(k-1)|$. Hua $[4 ; 5 ; 6]$ has shown that it is often helpful to consider the averages $n^{-1} \sum_{m=0}^{n} S(m)$. In this paper we consider some further developments of this idea.

1. Preliminaries. We recall $[7$, pp. $483-486,492-494]$ that if $\chi$ is a primitive residue character $\bmod k$ and if $\tau(\chi)=\sum_{n=1}^{k} \chi(n) e^{2 \pi i n / k}$, then $|\tau(\chi)|=k^{1 / 2}$ and

$$
\sum_{n=1}^{k} \chi(n) e^{2 \pi i m n / k}=\bar{\chi}(m) \tau(\chi)
$$

for any integer $m, \bar{\chi}$ being the complex conjugate of $\chi$.

Presented to the Society, April 30, 1949; received by the editors September 23, 1949.

${ }^{1}$ For the basic facts about residue characters see [7, pp. 401-414, 478-494]. Numbers in brackets refer to the bibliography. 
The function $S(k x)$ is of bounded variation and period unity, and as such has an everywhere convergent Fourier series. It may be deduced from (1) (cf. [13, pp. 23-24] and [8, pp. 81-82]) that this Fourier series is

$$
A(\chi)-\frac{\tau(\chi)}{2 \pi i} \sum_{m \neq 0} \frac{\bar{\chi}(m)}{m} e^{-2 \pi i m x},
$$

where $m$ runs over the positive and negative integers and

$$
A(\chi)=\int_{0}^{1} S(k x) d x=\frac{1}{k} \sum_{m=0}^{k-1} S(m) .
$$

Thus if we define a function $S^{*}(y)$ as $S(y)$ if $y$ is not an integer and as $S(y)-\chi(y) / 2$ if $y$ is an integer, we have

$$
S^{*}(k x)= \begin{cases}A(\chi)+\frac{\tau(\chi)}{\pi} \sum_{n=1}^{\infty} \frac{\bar{\chi}(n)}{n} \sin 2 \pi n x \text { if } & \chi(-1)=1 \\ A(\chi)-\frac{\tau(\chi)}{\pi i} \sum_{n=1}^{\infty} \frac{\bar{\chi}(n)}{n} \cos 2 \pi n x & \text { if } \quad \chi(-1)=-1\end{cases}
$$

for all non-negative $x$.

2. Another proof of Paley's $\Omega$-result for $S(n)$. If we put $x=0$ in (4) we get a formula for the arithmetic mean $A(\chi)$ of $S(0), \cdots$, $S(k-1)$, namely

$$
A(\chi)= \begin{cases}0 & \text { if } \chi(-1)=1 \\ \frac{\tau(\chi)}{\pi i} L(1, \bar{\chi}) & \text { if } \chi(-1)=-1,\end{cases}
$$

where $L\left(s, \bar{\chi}=\sum_{n=1}^{\infty} \bar{\chi}(n) n^{-\triangleleft}\right.$ for $R(s)>0$. The second half of formula (5) can of course be proved by the method used in [9] to prove the second part of Satz 217 (of which the second half of (5) is a generalization), but some use of Fourier series is essential. ${ }^{2}$

The second part of (5) enables us, when $\chi(-1)=-1$, to use information about the order of magnitude of $L(1, \bar{\chi})$ to get information about the order of magnitude of $A(\chi)$. For example, a slight change in the argument of [2] shows that for real primitive $\chi$ with $\chi(-1)=-1$ we have

$$
\limsup _{k \rightarrow \infty} \frac{L(1, \chi)}{\log \log k} \geqq e^{\gamma}
$$

2 Naturally (5) can be proved in a roundabout way by showing that both sides are equal to $L(0, x)$. The argument indicated here essentially stems from Minkowski (cf. $[13$, p. 26]). 
$\gamma$ being Euler's constant. (The lim sup is unambiguous, since for each $k$ there is at most one real primitive $\chi$ with $\chi(-1)=-1$.) Now $\tau(\chi)=i k^{1 / 2}$ for real primitive $\chi$ with $\chi(-1)=-1[9$, Satz 215] and thus (6) gives the following result.

THEOREM 1. If $\chi$ runs through all real primitive characters with $\chi(-1)=-1$ in order of increasing size of the modulus $k$ of $\chi$ and if $A(\chi)$ is as defined in (3), then

$$
\limsup _{k \rightarrow \infty} \frac{A(\chi)}{k^{1 / 2} \log \log k} \geqq \frac{e^{\gamma}}{\pi} .
$$

This theorem is a slightly stronger form of Paley's result (see [12; $10 ; 1])$ that if $\chi$ is a primitive character modulo $k$, then $M(\chi)$ $=\Omega\left(k^{1 / 2} \log \log k\right)$, where $M(\chi)$ is the maximum of $|S(0)|, \cdots$, $|S(k-1)|$; for our theorem shows that this $\Omega$-result is true even for the arithmetic mean of $S(0), \cdots, S(k-1)$.

We remark in passing that Parseval's formula applied to (2) or (4) gives

$$
\begin{aligned}
\frac{1}{k} \sum_{m=0}^{k-1}|S(m)-A(\chi)|^{2} & =\int_{0}^{1}|S(k x)-A(\chi)|^{2} d x \\
& =\frac{k}{2 \pi^{2}} \sum_{n=1}^{\infty} \frac{1}{n^{2}}=\frac{k}{12} \prod_{p \mid k}\left(1-\frac{1}{p^{2}}\right),
\end{aligned}
$$

where the ' indicates summation over the positive integers relatively prime to $k$. Alternatively we have, in view of (5),

$\frac{1}{k} \sum_{m=0}^{k-1}|S(m)|^{2}= \begin{cases}\frac{k}{2 \pi^{2}} \sum_{n=1}^{\infty} \frac{1}{n^{2}} & \text { if } x(-1)=1 \\ \frac{k}{\pi^{2}}\left(\frac{1}{2} \sum_{n=1}^{\infty} \frac{1}{n^{2}}+|L(1, x)|^{2}\right) & \text { if } x(-1)=-1 .\end{cases}$

3. Estimation of certain partial averages of $S(n)$. We saw above that if $\chi$ is a primitive character with $\chi(-1)=1$, then the arithmetic mean of the numbers $S(0), \cdots, S(k-1)$ is zero. Hence in this case we should expect a fairly good estimate of the arithmetic mean of $S(0), \cdots, S(n-1)$ for $n<k$. And in fact Hua $[4 ; 5 ; 6]$ has proved that we have

$$
\left|\frac{1}{n} \sum_{m=0}^{n-1} S(m)\right| \leqq \frac{1}{2}\left(k^{1 / 2}-\frac{n}{k^{1 / 2}}\right) \quad \text { for } \quad 0 \leqq n \leqq k, \chi(-1)=1 .
$$

If $\chi$ is a primitive character with $\chi(-1)=-1$, such a neat result 
cannot be expected, since the arithmetic mean of $S(0), \cdots, S(k-1)$ is not zero. However we prove the following result, which is valuable for $n$ not too small relative to $k$, especially if $|L(1, \chi)|$ is large.

TheOREM 2. If $\chi$ is a primitive character modulo $k$ with $\chi(-1)=-1$ and if $\alpha k \leqq n \leqq k$, where $\alpha$ is a number between 0 and 1 , then

$$
\left|\frac{1}{n} \sum_{m=0}^{n-1} S(m)-\frac{\tau(\chi)}{\pi i} L(1, \bar{x})\right|<\left(2.1+\frac{\pi}{4} \log \frac{1}{\alpha}\right) k^{1 / 2} .
$$

Proof. Put $q=[k / 2], \rho=e^{2 \pi i / k}$. Then by (1) we have

$$
\begin{aligned}
\tau(\bar{\chi}) S(m)= & \sum_{l=0}^{m} \chi(l) \tau(\bar{\chi})=\sum_{l=0}^{m} \sum_{h=-q}^{q} \bar{\chi}(h) \rho^{h l} \\
= & \sum_{h=1}^{q} \bar{\chi}(h) \sum_{l=0}^{m}\left(\rho^{h l}-\rho^{-h l}\right) \\
= & \sum_{h=1}^{q} \bar{\chi}(h) \frac{\rho^{h(m+1)}-1-\rho^{h}+\rho^{-h m}}{\rho^{h}-1} \\
= & \sum_{h=1}^{q} \bar{\chi}(h) \frac{\rho^{h(m+1)}+\rho^{-h m}}{\rho^{h}-1} \\
& +i \sum_{h=1}^{q} \bar{\chi}(h) \cot \frac{\pi h}{k} .
\end{aligned}
$$

Hence

$$
\begin{aligned}
\frac{\tau(\bar{\chi})}{n} \sum_{m=0}^{n-1} S(m)= & \frac{1}{n} \sum_{h=1}^{q} \bar{\chi}(h) \frac{\rho^{h(n+1)}-\rho^{-h(n-1)}}{\left(\rho^{h}-1\right)^{2}} \\
& +i \sum_{h=1}^{q} \bar{\chi}(h) \cot \frac{\pi h}{k} \\
= & \frac{1}{2 i n} \sum_{h=1}^{q} \bar{\chi}(h) \frac{\sin (2 \pi h n / k)}{\sin ^{2}(\pi h / k)} \\
& +i \sum_{h=1}^{q} \bar{\chi}(h) \cot \frac{\pi h}{k} .
\end{aligned}
$$

Now $\tau(\chi) \tau(\bar{\chi})=-\tau(\chi) \overline{\tau(\chi)}=-k$ for $\chi(-1)=-1$ and thus

$$
\begin{aligned}
\tau(\bar{\chi})\left\{\frac{1}{n} \sum_{m=0}^{n-1} S(m)-\right. & \left.\frac{\tau(\chi)}{\pi i} L(1, \bar{\chi})\right\} \\
& =\frac{\tau(\bar{\chi})}{n} \sum_{m=0}^{n-1} S(m)-\frac{i k}{\pi} L(1, \bar{\chi})
\end{aligned}
$$




$$
\begin{aligned}
= & \frac{1}{2 i n} \sum_{h=1}^{q} \bar{\chi}(h) \frac{\sin (2 \pi h n / k)}{\sin ^{2}(\pi h / k)} \\
& +i \sum_{h=1}^{q} \bar{\chi}(h)\left(\cot \frac{\pi h}{k}-\frac{1}{\pi h / k}\right) \\
& -\frac{i k}{\pi} \sum_{h=q+1}^{\infty} \frac{\bar{x}(h)}{h}=R_{1}+R_{2}+R_{3},
\end{aligned}
$$

say. If we put $S_{1}(h)=\sum_{n=q+1}^{n} \bar{\chi}(n)$ and use the fact that $\left|S_{1}(h)\right|<2-k$, we have

$$
\begin{aligned}
\left|R_{3}\right| & =\left|\frac{k}{\pi} \sum_{h=q+1}^{\infty} \frac{S_{1}(h)}{h(h+1)}\right| \leqq \frac{2^{-1} k^{2}}{\pi} \sum_{h=q+1}^{\infty} \frac{1}{h(h+1)} \\
& =\frac{2^{-1} k^{2}}{\pi(q+1)} \leqq \frac{k}{\pi} .
\end{aligned}
$$

Using the fact that $0<x^{-1}-\cot x \leqq 2 / \pi$ for $0<x \leqq \pi / 2$, we find

$$
\left|R_{2}\right| \leqq \frac{2}{\pi} q \leqq \frac{1}{\pi} k .
$$

We divide the summation in $R_{1}$ into two parts, namely $1 \leqq h$ $\leqq\left[\alpha^{-1}+1\right]$ and $\left[\alpha^{-1}+2\right] \leqq h \leqq q$. In the first part we use $\sin (2 \pi h n / k)$ $\leqq 2 \pi h n / k$, in the second part we use $\sin (2 \pi h n / k) \leqq 1$, and in both parts we use $\sin (\pi h / k) \geqq 2 h / k$. Thus

$$
\begin{aligned}
\left|R_{1}\right| & \leqq \frac{1}{2 n} \sum_{h=1}^{[\alpha-1+1]} \frac{2 \pi h n / k}{4 h^{2} / k^{2}}+\frac{1}{2 n} \sum_{h=\left[\alpha^{-1}+2\right]}^{q} \frac{1}{4 h^{2} / k^{2}} \\
& \leqq \frac{\pi}{4} k \sum_{h=1}^{[\alpha-1+1]} \frac{1}{h}+\frac{k^{2}}{8 n} \sum_{h=\left[\alpha^{-1}+2\right]}^{\infty} \frac{1}{h^{2}} \\
& \leqq \frac{\pi}{4} k\left(\frac{3}{2}+\log \frac{1}{\alpha}\right)+\frac{k^{2}}{8 n} \alpha \\
& \leqq\left(\frac{3 \pi}{8}+\frac{\pi}{4} \log \frac{1}{\alpha}+\frac{1}{8}\right) k .
\end{aligned}
$$

Combining (8), (9), (10), and (11), we get our theorem.

4. A theorem of Davenport. Davenport [3] has proved that if $s$ is a fixed complex number with $0<\sigma=R(s)<1$, then for any primitive $\chi$ we have $|L(s, \chi)| \leqq C k^{(1-\sigma) / 2}$ where $C$ is a constant depending on $s$. In this section we show how Hua's inequality (7) gives a very simple proof of this result in the case $\chi(-1)=1$, with a specific value of the 
constant. Unfortunately our Theorem 2 is too weak to give Davenport's result for the case $\chi(-1)=-1$.

Theorem 3. If $\chi$ is a primitive character with $\chi(-1)=1$ and if $0<\sigma=R(s)<1$, then

$$
|L(s, \chi)| \leqq \frac{|s(s+1)|}{\sigma(1-\sigma)} k^{(1-\sigma) / 2} .
$$

Proof. Put $T(n)=\sum_{m=0}^{n} S(m)$. Then we get by two partial summations

$$
\begin{aligned}
L(s, \chi) & =\sum_{n=1}^{\infty} \frac{\chi(n)}{n^{s}}=\sum_{n=1}^{\infty} S(n) s \int_{0}^{1} \frac{d y}{(n+y)^{s+1}} \\
& =\sum_{n=1}^{\infty} T(n) s(s+1) \int_{0}^{1} \int_{0}^{1} \frac{d x d y}{(n+x+y)^{s+2}} .
\end{aligned}
$$

For $n \leqq\left[k^{1 / 2}\right]$ clearly $|T(n)| \leqq \sum_{m=0}^{n} m \leqq n^{2}$, while for $n \geqq\left[k^{1 / 2}+1\right]$ we have by (7)

$$
|T(n)| \leqq 2^{-1} k^{1 / 2}\left\{n+1-(n+1)^{2} / k\right\} \leqq 2^{-1} k^{1 / 2} n .
$$

Thus

$$
\begin{aligned}
|L(s, x)| & \leqq|s(s+1)| \sum_{n=1}^{\infty} \frac{|T(n)|}{n^{\sigma+2}} \\
& =|s(s+1)|\left\{\sum_{n=1}^{\left[k^{1 / 2}\right]} \frac{1}{n^{\sigma}}+\sum_{n=\left[k^{1 / 2}\right]+1}^{\infty} \frac{2^{-1} k^{1 / 2}}{n^{\sigma+1}}\right\} \\
& \leqq|s(s+1)|\left\{1+\int_{1}^{\left[k^{1 / 2}\right]} \frac{d x}{x^{\sigma}}+2^{-1} k^{1 / 2} \int_{\left[k^{1 / 2}\right]}^{\infty} \frac{d x}{x^{\sigma+1}}\right\} \\
& \leqq|s(s+1)|\left\{1+\frac{k^{(1-\sigma) / 2}-1}{1-\sigma}+\frac{2^{-1} k^{1 / 2}}{\sigma\left[k^{1 / 2}\right] \sigma}\right\} \\
& \leqq|s(s+1)|\left\{\frac{k^{(1-\sigma) / 2}}{1-\sigma}+\frac{k^{(1-\sigma) / 2}}{\sigma}\right\}=\frac{|s(s+1)|}{\sigma(1-\sigma)} k^{(1-\sigma) / 2},
\end{aligned}
$$

which proves our theorem.

\section{BIBLIOGRAPHY}

1. S. Chowla, On the k-analogue of a result in the theory of the Riemann zeta function, Math. Zeit. vol. 38 (1934) pp. 483-487.

2. - On the class-number of the corpus $P\left((-k)^{1 / 2}\right)$, Proceedings of the National Institute of Sciences, India vol. 13 (1947) pp. 197-200. 
3. H. Davenport, On Dirichlet's L-functions, J. London Math. Soc. vol. 6 (1931) pp. 198-202.

4. L. K. Hua, On character sums, Science Record. Academia Sinica vol. 1 (1942) pp. 21-23.

5. - On the least primitive root of a prime, Bull. Amer. Math. Soc. vol. 48 (1942) pp. 726-730.

6. - On the least solution of Pell's equation, Bull. Amer. Math. Soc. vol. 48 (1942) pp. 731-735.

7. E. Landau, Handbuch der Lehre von der Verteilung der Primzahlen, vol. 1, Leipzig, 1909.

8. - Abschätzungen von Charaktersummen, Einheiten, und Klassenzahlen, Nachr. Ges. Wiss. Göttingen (1918) pp. 79-97.

9. — Vorlesungen uber Zahlentheorie, vol. 1, Leipzig, 1927. 32.

10. —_ Der Paleysche Satz ilber Charaktere, Math. Zeit. vol. 37 (1933) pp. 28-

11. U. V. Linnik and A. A. Renyi, On certain hypotheses in the theory of Dirichlet characters, Bull. Acad. Sci. URSS Sér. Math. vol. 11 (1947) pp. 539-546.

12. R. E. A. C. Paley, A theorem on characters, J. London Math. Soc. vol. 7 (1932) pp. $28-32$.

13. G. Pólya, Über die Verteilung der quadratischen Reste und Nichtreste, Nachr. Ges. Wiss. Göttingen (1918) pp. 21-29.

14. J. Schur, Einige Bemerkungen zu den vorstehenden Arbeit des Herrn G. Polya, Nachr. Ges. Wiss. Göttingen (1918) pp. 30-36.

INSTITUTE fOR AdVANCEd STUdy 